\title{
Implementation of the scanning laser Doppler vibrometer combined with a light-weight pneumatic artificial muscle actuator for the modal analysis of a civil structure
}

\author{
K. Deckers ${ }^{\mathrm{a}, *}$, P. Guillaume ${ }^{\mathrm{a}}$, C. Vuye $\mathrm{V}^{\mathrm{a}, \mathrm{b}}$ and D. Lefeber ${ }^{\mathrm{a}}$ \\ ${ }^{a}$ Acoustics and Vibration Research Group, Department of Mechanical Engineering, Vrije Universiteit Brussel, \\ Pleinlaan 2, B-1050 Brussels, Belgium \\ ${ }^{\mathrm{b}}$ Department of Applied Science and Technology, Artesis Hogeschool Antwerpen, Paardenmarkt 92, B-2000 \\ Antwerp, Belgium
}

\begin{abstract}
The identification of the modal parameters of bridges and other large civil constructions has become an important research issue. Different approaches have been proposed depending on the excitation used: ambient excitations (due to wind, traffic, ...) or artificial excitations (e.g. impact test with heavy drop weights). In practice it turns out that not all modes are well excited by the ambient forces. Hence the application of an artificial actuator is advisable. The problem is that larger constructions often require large and heavy excitation devices, which are hard to manipulate. Another difficulty encountered in performing a modal analysis on large civil constructions is the necessity for a large number of high sensitivity sensors. Consequently a large number of cables has to be installed resulting in a large setup time.

This paper is a proof-of-concept which demonstrates the possibility of using lightweight Pneumatic Artificial Muscles combined with the scanning laser Doppler vibrometer to perform a modal analysis on a civil structure. This combination allows for an important reduction in setup time and allows for sine testing as well as the application of broadband signals such as periodic chirps, true noise or multisines.
\end{abstract}

Keywords: Modal analysis, scanning laser Doppler vibrometer, pneumatic artificial muscle

\section{Introduction}

Different approaches to perform a modal analysis on civil constructions such as bridges are commonly in practice [1-12]. Depending on the excitation used these methods are divided into Experimental Modal Analysis (EMA) or Operational Modal Analysis (OMA).

Experimental Modal Analysis identification methods and procedures are forced excitation tests where the applied forces can be measured together with the response of the structure (e.g. accelerations). If applicable, EMA is the preferred approach as it usually results in better modal model estimates including scaled mode shapes. Properly applying an excitation device, however, is in practice often problematic when the tested structure is large.

In applications where it is rather difficult to apply an artificial force Operational Modal Analysis can be used. During OMA, the very low levels of dynamic response introduced by wind and/or traffic are measured with high sensitivity transducers. In such cases, it is practically impossible to measure the ambient excitations, and

\footnotetext{
*Corresponding author. Tel.: +32 26292324; Fax: +32 26292865; E-mail: kristel.deckers@ vub.ac.be.
} 
Table 1

Comparison of excitation sources

\begin{tabular}{lccccc}
\hline Criterion & Ambient & Shaker & Drop weight & Hammer & PAM \\
\hline Investment cost & ++ & -- & + & + & + \\
Installation cost & ++ & -- & + & ++ & - \\
Power consumption & ++ & -- & + & ++ & 0 \\
Low-frequent excitation & ++ & 0 & + & ++ & 0 \\
High-frequent excitation & - & ++ & + & + & + \\
Modal scaling factors & -- & ++ & ++ & + & + \\
Controlled amplitude & -- & ++ & ++ & 0 & ++ \\
Controlled signal & -- & ++ & 0 & - & ++ \\
Maximum amplitude & - & ++ & ++ & - & 0 \\
\hline
\end{tabular}

consequently, the responses are the only signals that can be measured. Traditionally, one assumes that the outputs are the results of a stochastic process with white noise sources as inputs [1,2]. Because the ambient forces are unknown, only unscaled modal model estimates can be obtained. Additional measurements are required to obtain a scaled modal model as explained in [4]. Examples of structures successfully tested with OMA are the Heritage Court Tower (Vancouver, Canada) and the Vasco da Gama Bridge [3].

Since the ambient excitation can not be controlled and the excitation level can be small, it is possible that some modes of vibration are not (sufficiently) excited. When this is the case, exciters are required to excite the system in all frequencies of interest. In small and medium size structures, the excitation can be induced by an impulse hammer. This device has the advantage of providing a wide-band input, but as a drawback, also has a relatively low frequency resolution (see [3] for more information regarding excitation techniques in modal analysis). Controlled excitation of large civil engineering structures is more complex and introduces the necessity of heavy excitation equipment. Examples of equipment useful for this kind of experiments are large electrodynamic shakers, eccentric mass vibrators or servo-hydraulic shakers. Some laboratories have special impulse devices specifically designed to excite bridges (also see [3]).

To overcome some of the problems mentioned above the Pneumatic Artificial Muscle (PAM) is considered in this research as actuator in an EMA-setup, see Section 2.

Recently, it was demonstrated that lightweight PAMs can be used to excite civil constructions. In [5] the PAM is applied as an additional actuator to the ambient traffic and wind excitation. It is shown that the PAM can aide in identifying eigenfrequencies and mode shapes that are badly excited by ambient excitation alone. In [6] this actuator is compared to other more commonly applied actuation methods. There are several different comparison criteria like e.g. application cost, excitation abilities, signal control, etc. Table 1 provides a recapitulation of the most important comparison criteria for several excitation sources (Table provided in [6]). Their properties are assessed from very favorable (++) Over neutral (0) to very unfavorable (- ). The study leads to the conclusion that:

"If a specific signal is needed, such as a multisine, a (periodic/burst) random signal, or a swept sine, and a maximum force amplitude of several $\mathrm{kN}$ is sufficient, the use of a pneumatic artificial muscle (PAM) should be considered as an alternative for a shaker, since its investment cost is smaller."

The difficulty encountered in performing the measurement of [5] was the necessity for a large number of high sensitivity sensors, which have to be placed along the length of the tested structure. The deployment of these sensors is often met with practical problems concerning the sensors themselves and the long cables that are necessary to connect them:

- the risk of sensors failing during acquisition,

- the time consuming placement of the sensors which is often much longer than the actual measurement time,

- the fact that it is not always easy to connect the sensors, especially in places which are difficult to access,

- and last but not least, the result of using a limited number of sensors is a low spatial resolution.

These limitations can be avoided by using the Scanning Laser Doppler Vibrometer (SLDV) which is equipped with automatic, highly sensitive, non-contact scanning capabilities. It allows for the responses to be captured at various locations on a structure. These features allow for vibration measurements with higher spatial resolution that can only be matched by conventional measurement methods using a lot of high sensitivity transducers. Examples of measurements performed on larger structures by means of the SLDV can be found in [7,8]. Where issues arise 


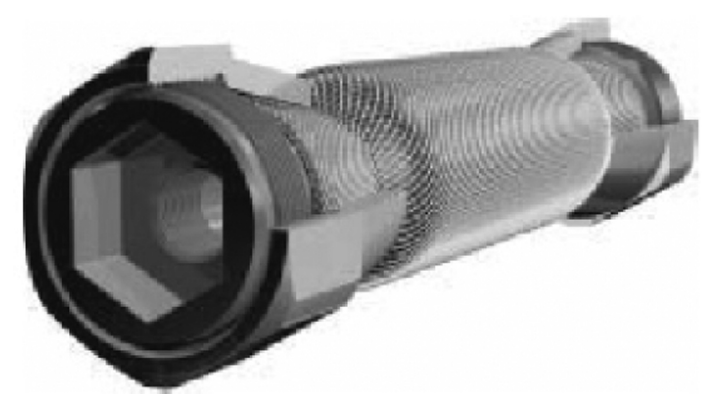

Fig. 1. Design of the Fluidic Muscle DMSP by Festo AG and Co. KG.

concerning the reflection of the laser beam $[7,8]$ introduce a spatial vibration measurement system that searches the vicinity of an insufficient measurement point for the optimum point with maximum reflected laser beam quality.

When measuring over large distances, it can be difficult to obtain adequate reflection. Recently a remote sensing vibrometer (RSV-150) with higher power was tested by the KU Leuven and Polytec [9], where two vibration measurements over distances of approximately $81 \mathrm{~m}$ and $211 \mathrm{~m}$ are performed. Some of the difficulties encountered during these measurements are reflection problems, weather and other environmental disturbances. Most of these difficulties can be dealt with by visually inspecting the measured data and selecting the appropriate data sections for the identification algorithm.

Experiments have been performed using the SLDV for the structural parameter identification of a structural member in different measurement conditions in [10]. [11] investigates the application of the SLDV for structural health monitoring and covers the issues of data acquisition, system identification and structural damage detection. Other advantages of the SLDV system are reduction of the measurement time and possibility for the measurement of structural elements located in a difficult position or a hazardous environment since the data acquisition is possible without contact [12].

Together with the SLDV the PAM could form a useful alternative for the heavy equipment commonly used in modal analysis. This paper therefore considers the case study of using the SLDV together with the light-weight Pneumatic Artificial Muscle to perform a modal analysis, as a proof of concept, on the ceiling of a large laboratory. For that reason, the aim of the paper is twofold:

- to show that a PAM can be used to excite large heavy constructions. Moreover, by using a proportional valve, arbitrary forces can be applied. Actuators that are used nowadays to apply an arbitrary excitation are quite heavy and difficult to install, resulting in a very long setup time. In short, the PAM can be viewed as a kind of lightweight shaker that can apply very large forces.

- to show that the implementation of the SLDV to perform the vibration measurements is more practical than using accelerometers. After all, the measurement locations are not always easily accessible and very long cables are required between the high sensitivity transducers and the acquisition system. This implies that the setup time can be very long.

\section{Concept of the Pneumatic Artificial Muscle}

The Pneumatic Artificial Muscles, or PAMs, are generally applied in the field of robotics, to simulate human muscle behavior. These muscles are lightweight actuators that can apply several $\mathrm{kN}$ of pulling force, depending on their size. It is therefore very interesting to use them as actuators in applications other than robotics. In this paper a PAM is used to excite the roof structure of a large laboratory to show that a lightweight PAM can be used to excite larger civil structures without the necessity of the heavy equipment described in Section 1.

Different types of PAMs exist, e.g. the second generation Pleated Pneumatic Artificial Muscle (or PPAM), which was developed at the department of Robotics and Multibody Dynamics at the Vrije Universiteit Brussel [13], and the Fluidic Muscle DMSP developed by Festo AG AND Co.KG which is commercially available [14,15]. The muscle considered in this implementation is the commercially available Festo DMSP see Fig. 1. 

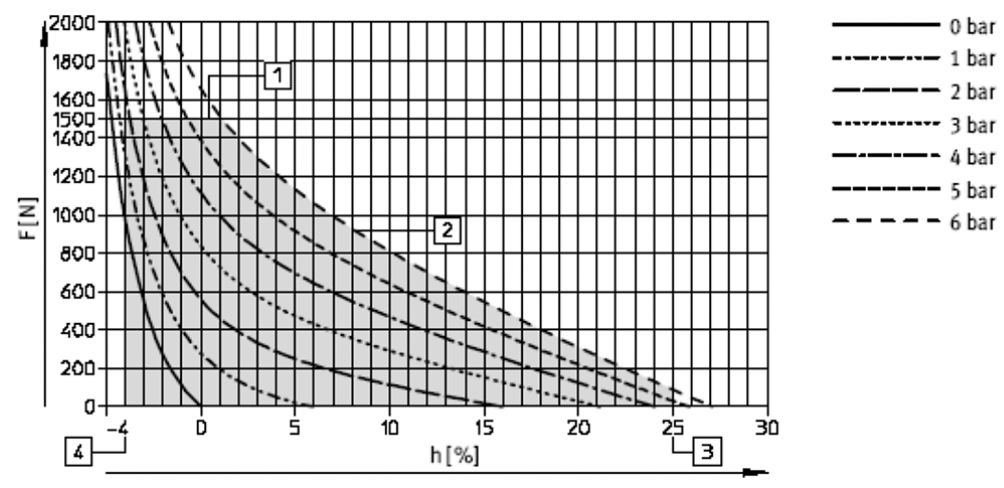

1 Min. theoretical forceat max. operating pressure

2. Max. operating pressure

3. Max. deformation

4 Max. pretensioning

Permissible operating range

Fig. 2. Pulling force versus contraction of the DMSP20 [15].

This actuator comes in different sizes and configurations. The basic concept lies in the combination of an impervious, flexible hose and a covering of woven fibres as tensile material in a rhomboidal mesh. This results in a three dimensional grid structure. The pressurized air flowing inwards changes the shape of the grid structure by expansion, thus generating a tensile force in the axial direction. The grid structure causes the muscle to shorten up as internal pressure is increased. This corresponds to a stroke of approximately 25 percent of the initial unloaded length.

Figure 2 shows the force-contraction relation of the PAM which was used during this research. At low contractions the allowed force is restricted to protect the pressed end fittings from damage. The force-contraction relation shown is that of a DMSP20 (this means that the contractible membrane's inner diameter is $20 \mathrm{~mm}$ ) which has a total nominal length of $28 \mathrm{~cm}$ and weighs less than $0.200 \mathrm{~kg}$. It can exert up to $1500 \mathrm{~N}$ of pulling force at a pressure of just $6 \mathrm{bar}$.

The muscle can only exert pulling forces, due to its design principle explained above. This will be taken into account when designing a test setup.

Another interesting property of the PAM to regard is the available bandwidth. The effective bandwidth depends on many factors like e.g. the amplitude of the input signal, contraction levels, the type of PAM considered, the available pressure, etc. For the DMSP20, used in this case study and with a setup which limits contraction levels, the determined bandwidth was about $15 \mathrm{~Hz}$. It should be noted that higher frequencies can be applied with the DMSP20, but the resulting amplitudes will be smaller.

\section{Experimental setup}

To determine whether the PAM can be used to perform a modal analysis on a civil structure, the ceiling of a large laboratory is excited as a proof of concept. Figure 3 shows the schematic representation of the experimental setup. Since the PAM can only exert tensile forces, it was decided to use kevlar cables of $6 \mathrm{~mm}$ diameter to transfer the applied forces to the ceiling structure. A simple clamping mechanism is used to make the connection to the joist itself (as is shown in Fig. 4). At the top a force sensor (a PCB222B ICP force sensor with sensitivity $0.1725 \mathrm{mV} / \mathrm{N}$ ) is placed to measure the actual transferred forces to the joist of the ceiling. At the bottom the muscle is attached to the wall just below the second floor to establish a fixed point.

The PAM is controlled through a proportional pressure valve (Kolvenbach KPS 3/6 valve) which converts $1 \mathrm{~V}$ of input signal to 1 bar of pressure at the output of the valve, for frequencies up to $20 \mathrm{~Hz}$. The valve itself is fed with a pressure vessel at 6 bar (see Fig. 5). This construction allows for a simple and perfect control of the force input signal and a perfect control of the input signal.

Note that a large $2 \mathrm{kN}$ shaker could be used to apply arbitrary excitations as well. The weight of such a shaker is much higher, however, and the connection to the ceiling would pose more difficulties.

The selected input signal is a periodic chirp between 0 and $10 \mathrm{~Hz}$, with an amplitude of $2 \mathrm{~V}$ and offset $3 \mathrm{~V}$. The offset is necessary to have a positive input signal for the valve and thus a positive pressure for the muscle resulting in the desired tensile forces with a measured peak-to-peak value of about $1 \mathrm{kN}$. 


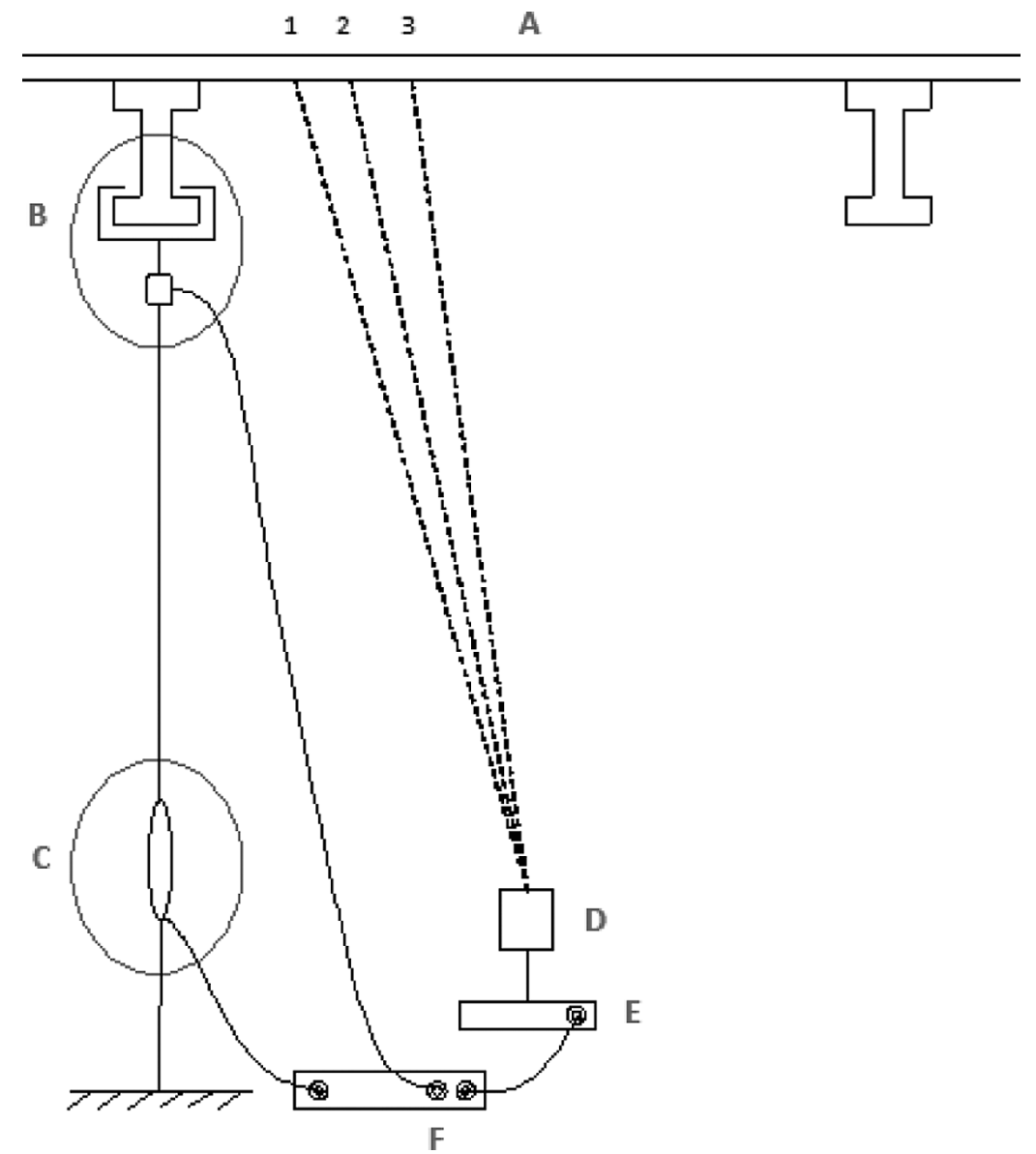

Fig. 3. Schematic representation of the measurement setup. A: Tested ceiling structure with scanned measurement grid $(1,2, \ldots, 72)$, B: Joist and its connection with the PAM (Fig. 4), C: PAM system (Fig. 5), D: Laser head, E: SLDV decoder, F:Computer with generator and acquisition system.

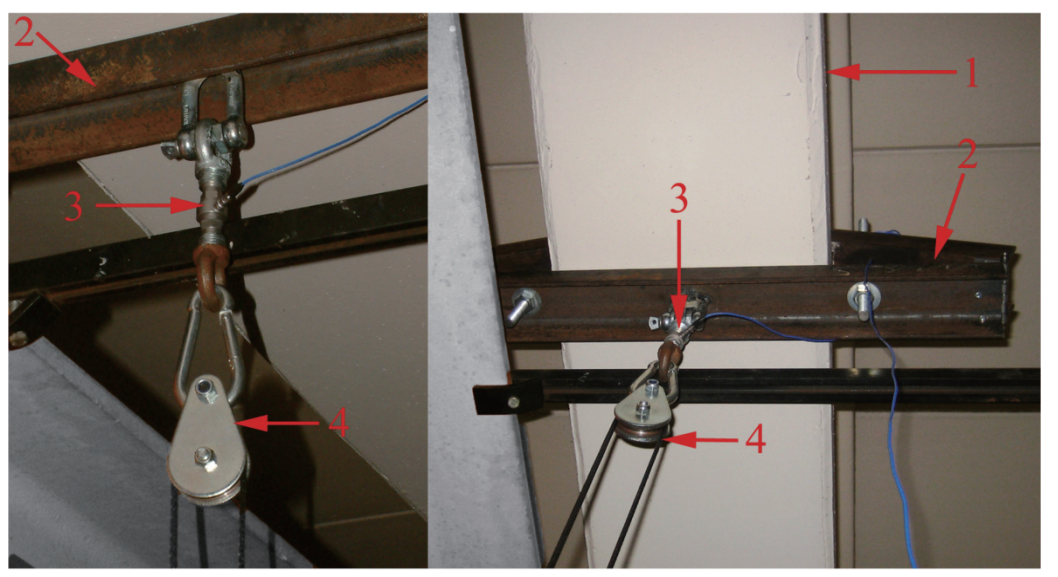

Fig. 4. Position of the force sensor and connection of the DMSP20 to the joist structure of the ceiling. 1: Joist of the ceiling, 2: Clamping mechanism, 3: Force cell, 4: Pulley connection with the kevlar cable. 


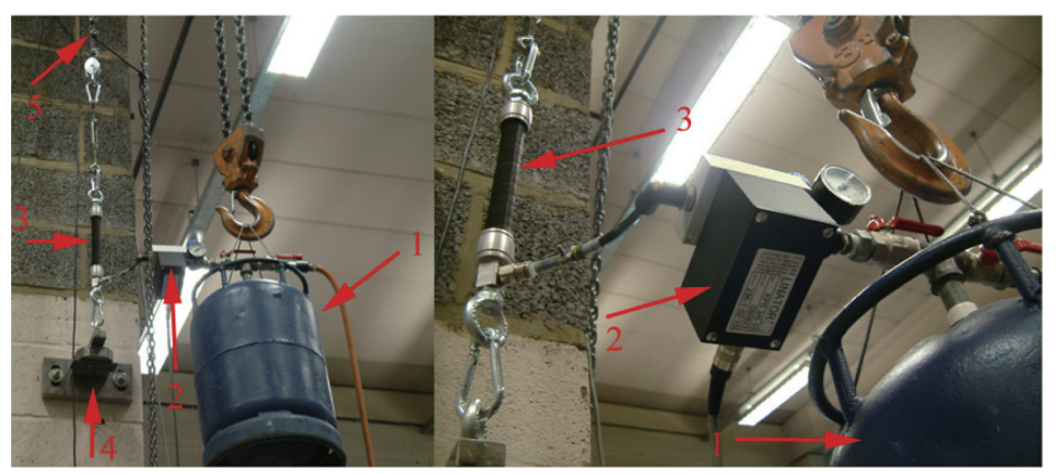

Fig. 5. Connection of the DMSP20 to the wall and setup of the proportional valve and pressure vessel. 1: Pressure vessel, 2: Proportional valve, 3: DMSP20, 4: Attachment to the wall, 5: Connection with the kevlar cable.

The data acquisition is performed using a SLDV (Polytec PSV400). A grid of 8 by 9 measurement points is selected in between two consecutive joists to ensure that the movement of only the ceiling panels is registered. The acquisition mode is set to FFT with a complex averaging of five periods. The bandwidth is set between 0 and $20 \mathrm{~Hz}$ since the spectral composition of the excitation signal is limited to $10 \mathrm{~Hz}$. The sampling frequency and measurement time per scanned point are $640 \mathrm{~Hz}$ and $25.6 \mathrm{~s}$ respectively, resulting in 6400 FFT lines and a spectral resolution of $39 \mathrm{mHz}$.

To compare the quality of the measurement with (EMA) and without (OMA) the use of the pneumatic actuator two measurements were performed. Because the acquisition is done using the SLDV, an accelerometer was mounted on the ceiling as a reference. This reference measurement is necessary for the OMA approach to obtain useful phase information and to cope with the non-stationarity of the ambient excitation (see [16]).

In this paper very basic analysis techniques are used because the focus is on the advantages of using a PAM in combination with a SLDV. For that reason only a basic analysis technique (peak-picking) is applied. Of course, the more advanced processing techniques (such as the OMAX approach $[17,18]$ ) can be used here too.

During the processing of the measurements with the PAM (periodic chirp excitation) the classical H1 estimator was used to derive the frequency response functions. This way the contribution of the ambient excitation to the response (which is very small in this case) has been eliminated by the averaging process.

\section{Experimental results}

Two consecutive measurements were performed on the ceiling of the laboratory: an EMA measurement to prove this simple setup can give excellent results and an OMA measurement that will serve as a reference for the technique most often used in modal analysis nowadays. In both cases the first three eigenfrequencies and their mode shapes are determined.

\subsection{EMA measurements}

When performing EMA measurements both the input and output signals are gathered. The measurements are synchronized with the periodic source signal. In every measurement point 3 periods of both input and output signal were acquired. The force signal depicted in Fig. 6 is measured at the clamping mechanism and will be considered as the input force to the tested system. This allows for detecting any discrepancies between the target signal sent to the proportional valve and the actually applied force. The nonlinear relation between contraction, fluid pressure and force output of the DMSP (reported in Fig. 2) as well as nonlinearities introduced by the elongation of the kevlar rope are responsible for these discrepancies. The nonlinearities introduce higher order frequency components in the actual excitation signal, as can be observed in Fig. 6. 


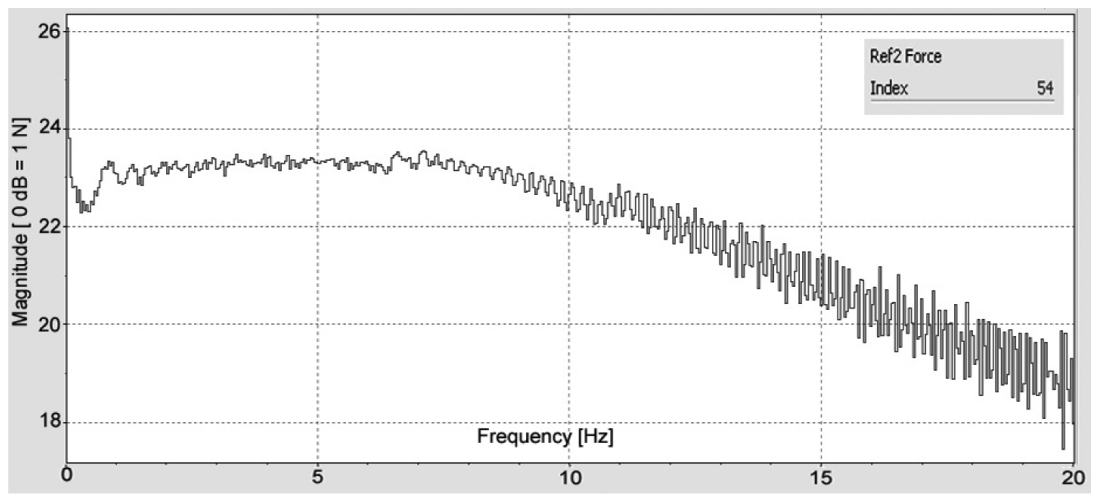

Fig. 6. Measured force applied to the joist.
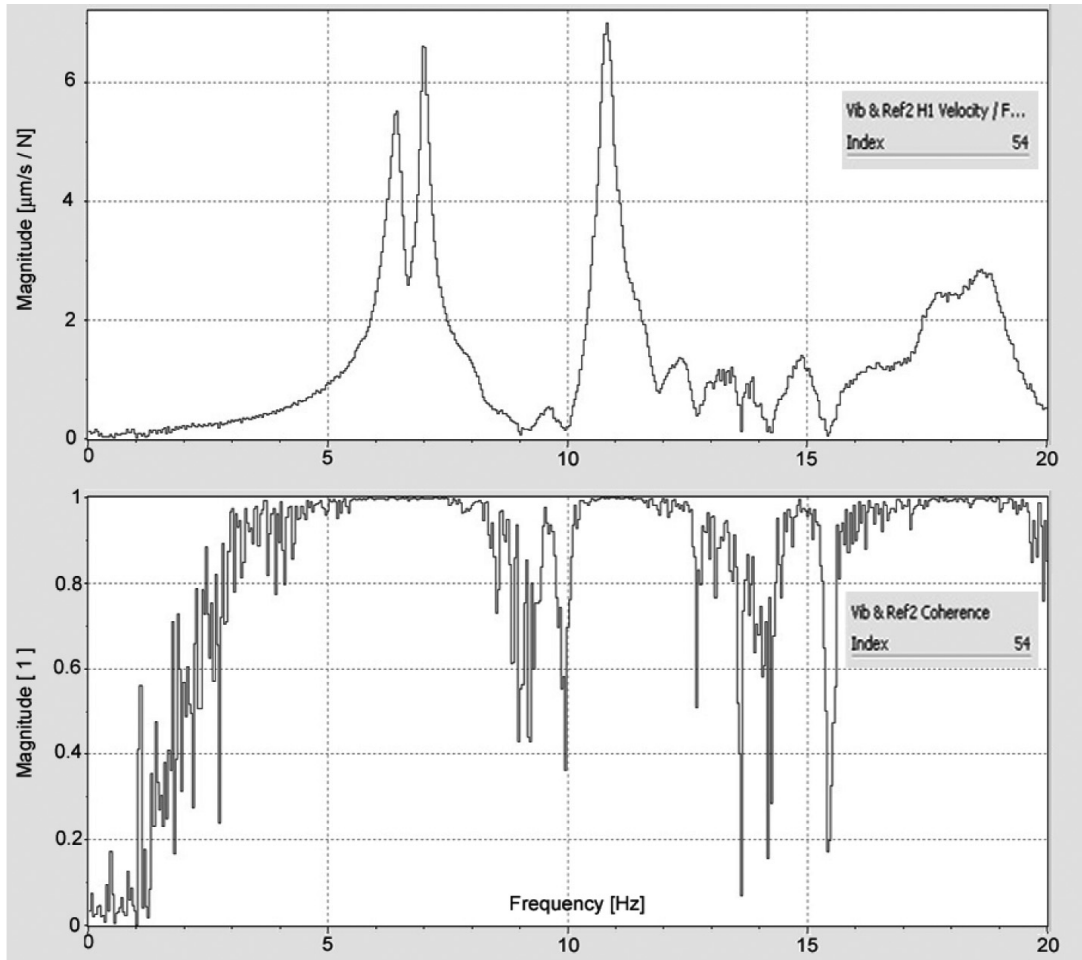

Fig. 7. Measured FRF in a selected measurement point and corresponding coherence function.

Note that Fig. 6 depicts the force spectrum. In the time domain the peak-to-peak value of the excitation level is about $1 \mathrm{kN}$ which is enough to drown out the ambient excitation. The ambient excitation can therefore be considered as noise which is eliminated by the averaging process.

The measured forces allow for calculating the FRF's between the outputs and the measured input. Fig. 7 shows one such FRF between the applied force and a chosen measurement point (point highlighted in the resulting mode shapes of Fig. 8). The coherence function shows that the correlation function is excellent in the resonance frequencies, resulting in the clear mode shapes of Fig. 8. The measured eigenfrequencies of the first three modes are listed in Table 2.

Since the nonlinearities resulted in excitation frequencies above $10 \mathrm{~Hz}$ (as was illustrated in Fig. 5), the third eigenfrequency of $10.78 \mathrm{~Hz}$ was excited well enough to be identified. We can therefore conclude that the excitation 
Table 2

Estimated eigenfrequencies

\begin{tabular}{lcc}
\hline & \multicolumn{2}{c}{ Eigenfrequency [Hz] } \\
\cline { 2 - 3 } Mode Number & EMA & OMA \\
\hline 1 & 6.4062 & 6.4453 \\
2 & 6.9922 & 6.9141 \\
3 & 10.7812 & 10.8594 \\
\hline
\end{tabular}

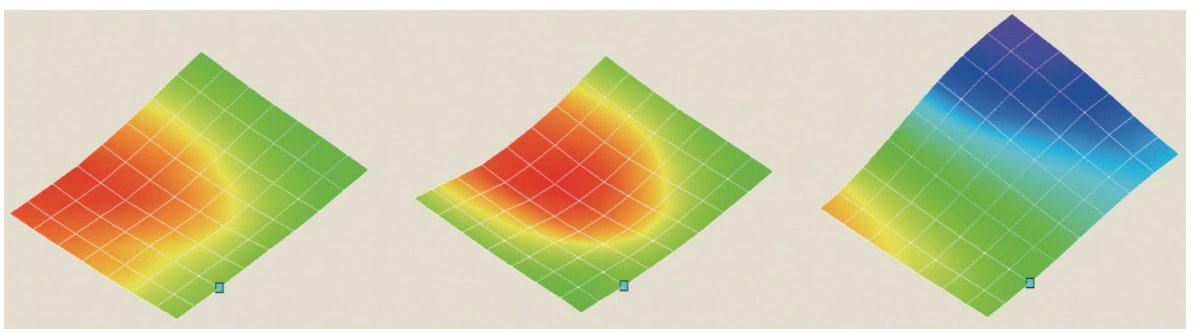

Fig. 8. Mode shapes of the first three eigenfrequencies of the ceiling determined with EMA.

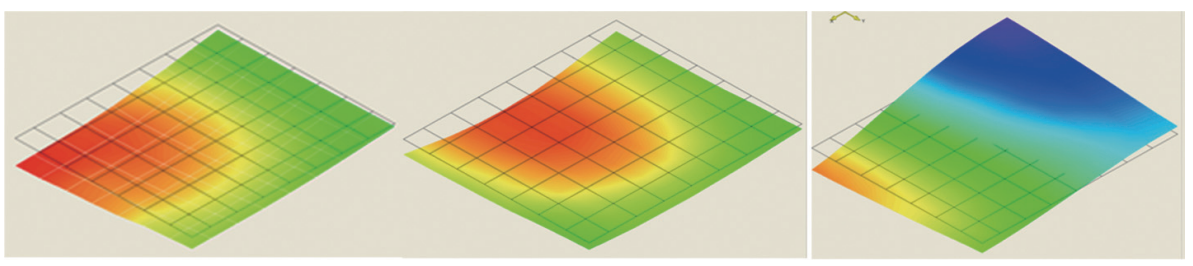

Fig. 9. Mode shapes determined during a fast scan with sine excitation.

by means of the DMSP20 was sufficient to properly excite and identify the first three modes of the ceiling. Because the excitation levels were measured, the resulting mode shapes are scaled.

The advantage of implementing the light-weight PAM actuator is the ease with which it can be attached to a structure. A combination of pulleys and kevlar rope make most places within reach. Moreover, it can apply forces even if it is not perfectly aligned, without harming the actuator. The cost of a PAM is also much lower than the cost of a shaker and it is less fragile, which allows for some trial and error in finding the most optimal attachment point.

The PAM, like a shaker, allows for choosing the input signal. In the EMA case previously discussed, a periodic chirp was applied. By changing the target signal for the proportional valve, any other signal within the range of about $15 \mathrm{~Hz}$ (the approximate bandwidth of the DMSP20 in this setup) can be applied. This is demonstrated by performing a sine test of the ceiling.

To perform a sine test, the frequencies of the sines to be applied have to be determined. Usually this is done by performing a single sweep and detecting the resonances by peak-picking. Next a sine is applied as input signal with a frequency that equals the first eigenfrequency. Then, a fast scan is executed, during which no averaging is implemented. The fast scan is repeated for the other two eigenfrequencies. The resulting mode shapes are shown in Fig. 9 and compare very nicely to the ones in Fig. 8. Since the input forces were measured, these mode shapes are scaled as well.

To validate the determined mode shapes in a quantitative manner, the MAC matrix of the mode shapes is calculated. Table 3 lists the MAC values of the MAC matrix for the EMA measurement with a swept sine input force signal. It should be noted that modes 1 and 2 have a high correlation, because only part of their mode shape was measured (a specific part of the ceiling was scanned and not the entire ceiling).

To compare both measurements (periodic chirp and sine), a cross MAC can also be determined. Table 4 list the MAC values of this cross matrix. Again the high values between mode shapes 1 and 2 result from the fact that the mode shapes are only partially identified. The cross MAC matrix confirms what has been visually established, the two measurement methods compare very nicely to one another. 
Table 3

MAC values for the periodic chirp measurements

\begin{tabular}{lccc}
\hline Mode Number & 1 & 2 & 3 \\
\hline 1 & 1 & 0.9244 & 0.0223 \\
2 & 0.9244 & 1 & 0.0143 \\
3 & 0.0223 & 0.0143 & 1 \\
\hline
\end{tabular}

Table 4

Cross MAC values for the periodic chirp (rows) and the sine (columns) measurements

\begin{tabular}{lccc}
\hline Mode Number & 1 & 2 & 3 \\
\hline 1 & 0.9774 & 0.9361 & 0.0608 \\
2 & 0.8789 & 0.9727 & 0.0360 \\
3 & 0.0537 & 0.0060 & 0.9554 \\
\hline
\end{tabular}

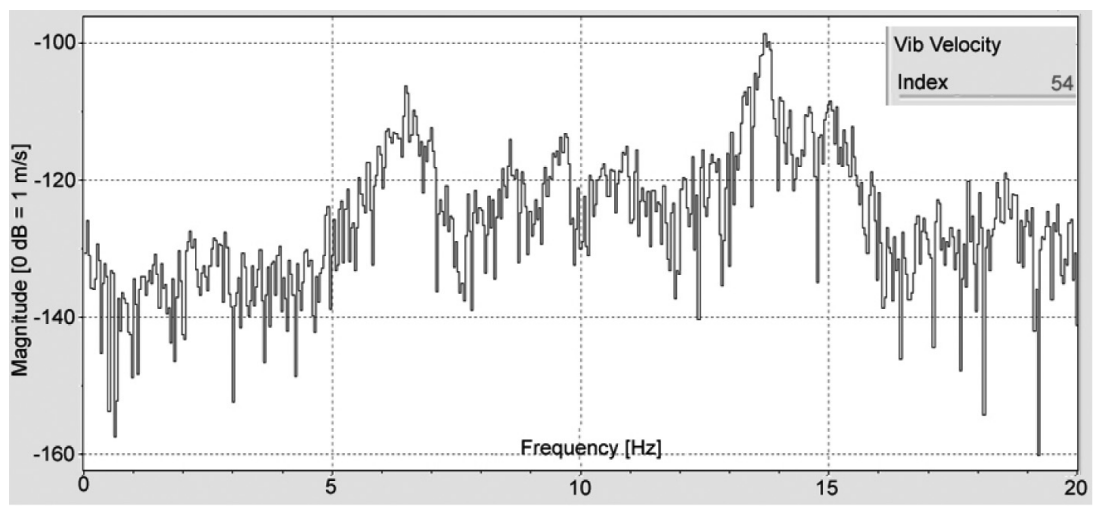

(a) OMA measurement

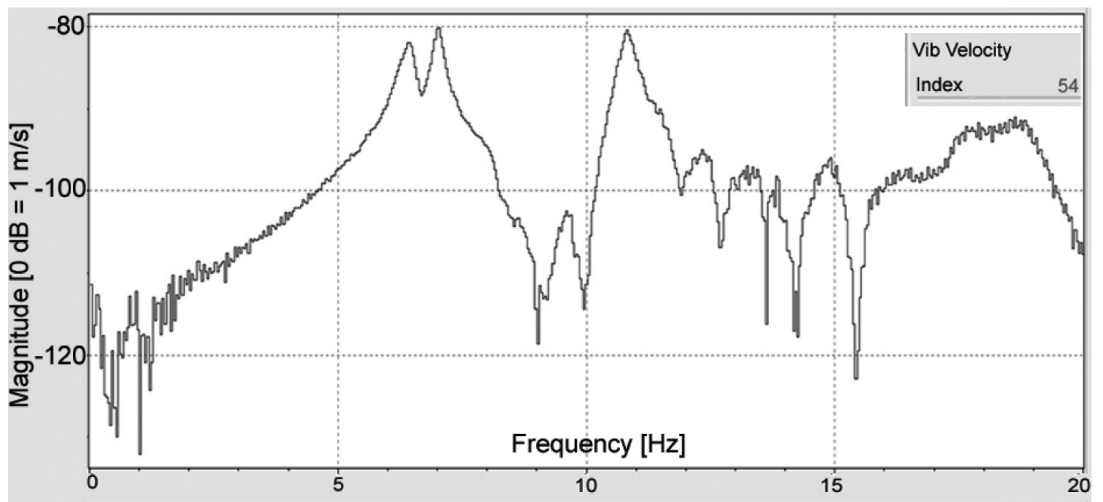

(b) EMA measurement

Fig. 10. Comparison of the vibrometer signal measured during OMA (top) and EMA (bottom), in measurement point 54.

\subsection{Experimental results of the OMA measurements}

As mentioned in Section 1, OMA doesn't use any artificial excitation, only the ambient excitation present during operational conditions is considered. This ambient excitation is considered to be white noise by the output-only identification algorithms. Figure 10 shows a frequency spectrum of the measured vibrometer signal caused by the ambient excitation (top) and the spectrum of the vibrometer signal measured during EMA (bottom). Comparing both figures shows that the EMA measurement results in vibration signals that are about ten times $(20 \mathrm{~dB})$ larger than the 


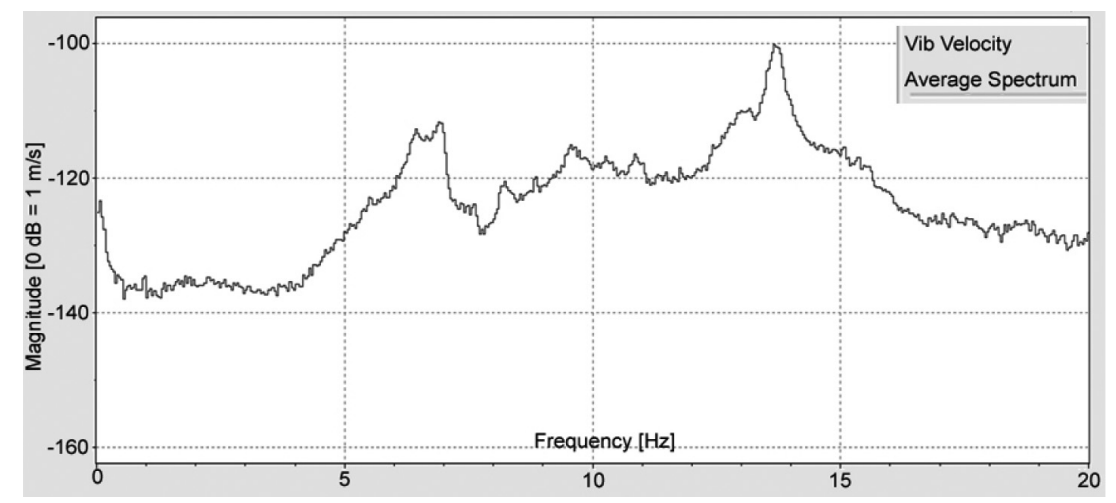

Fig. 11. Averaged vibrometer signal determined during the OMA measurement.

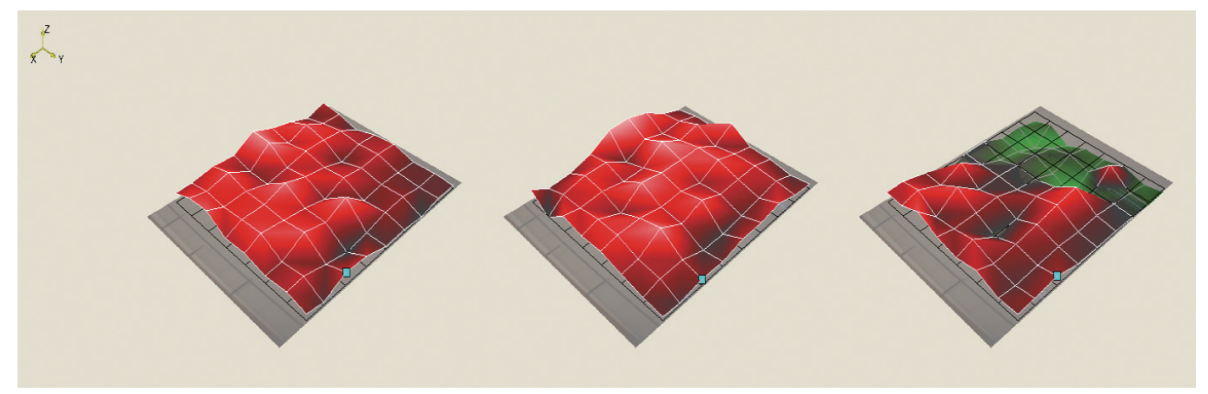

Fig. 12. Mode shapes of the first three eigenfrequencies of the ceiling determined with OMA.

vibrations measured during OMA. The assumptions that the ambient excitation could be considered as noise during the EMA measurement was therefore justified.

Since peak-picking with noisy data is not obvious, the average spectrum (Fig. 11) was used to determine the eigenfrequencies and mode shapes in the OMA case. The third mode does not appear in the spectrum because this mode is not well excited by the ambient excitation. The EMA measurements have shown the mode to exist, so the region between 10 and $11 \mathrm{HZ}$ was selected for these measurements as well. The resulting eigenfrequencies are listed in Table 2 and Fig. 12 shows their respective mode shapes. Evidently, these mode shapes resemble the ones found in Section 4.1, although they are not as well defined. We can therefore conclude that the ambient excitation was not sufficient to well excite the resonance frequencies.

\section{Conclusions}

In this paper a case study was performed to determine whether the PAM can be used in combination with the SLDV to improve the resonant frequency and mode shape estimates of civil structures. It is shown that the light-weight PAM could infuse enough energy into the structure to well excite and identify the mode shapes of the ceiling of a laboratory. The setup used is very simple but efficient and makes it possible to choose the excitation signal. The proposed setup can easily be modified to test other civil structures such as bridges or buildings.

The use of the SLDV allows for a high spatial resolution of the performed measurement without the necessity for a large number of high sensitivity senors. Moreover, the application of the SLDV ensures a minimization of the mass loading effect which would occur when applying a large number of sensors.

Compared to the traditionally performed OMA (without any artificial excitation), this EMA approach resulted in clear FRF's which made the modal analysis easy (peak-picking was used). The resulting mode shapes were far better than the ones determined without the muscle excitation. 


\section{Acknowledgments}

Research funded by a Ph.D grant of the Institute for the Promotion of Innovation through Science and Technology in Flanders (IWT-Vlaanderen). The authors also acknowledge the Flemish government (GOA-OPTIMech), the Fund for Scientific Research - Flanders (FWO), and the research council of the Vrije Universiteit Brussel (OZR) for their funding.

\section{References}

[1] B. Peeters and G. De Roeck, Stochastic system identification for operational modal analysis: A review, ASME Journal of Dynamic Systems, Measurement, and Control 123(4) (2001), 659-667.

[2] B. Cauberghe, Applied Frequency-domain System Identification in the Field of Experimental and Operational Modal Analysis. PhD Thesis, Vrije Universiteit Brussel, May 2004. http://phd.avrg.be.

[3] A. Cunha and E. Caetano, Experimental Modal Analysis of Civil Engineering Structures, Sound and Vibration 40(6) (June 2006), 12-20.

[4] E. Parloo, B. Cauberghe, F. Benedettini, R. Alaggio and P. Guillaume, Sensitivity-based Operational Mode Shape Normalization: Application to a Bridge, Mechanical Systems and Signal Processing 19(1) (January 2005), 43-55.

[5] K. Deckers, T. De Troyer, E. Reynders, P. Guillaume, D. Lefeber and G. De Roeck, Applicability of Low-weight Pneumatic Artificial Muscle Actuators in an OMAX Framework. Proceedings of ISMA 2008 International Conference on Noise and Vibration Engineering, September 2008, pp. 2445-2456.

[6] E. Reynders, D. Degrauwe, M. Schevenels, P. Van den Broeck, K. Deckers, P. Guillaume, F. Magalhas, E. Caetano and A. Cunha, OMAX Testing of a Bow-string and Stress-ribbon footbridge. Proceedings of ISMA 2008 International Conference on Noise and Vibration Engineering, September 2008, pp. 2549-2562.

[7] K. Kaito, M. Abe and Y. Fujino, An Experimental Modal analysis for RC Bridge Deckes Based on Non-contact Vibration Measurement, IMAC-Proceedings of the 19th International Modal Analysis Conference: A Conference on Structural Dynamics I and II (2001), 15611567.

[8] K. Kaito, M. Abe and Y. Fujino, Development of Non-contact Scanning Vibration Measurement System for Real-scale Structures, Structure and Infrastructure Engineering 1(3) (September 2005), 189-205.

[9] F. Percivale, T.T. Bui, L. He and G. De Roeck, Demonstration of Remote Sensing Vibrometer RSV-150 by Polytec: Data Processing and Modal analysis of Fabre's Totem and VRT Antenna Tower. Internal Report by the Katholieke Universiteit Leuven, Report BMW-2010-11, September 2010 .

[10] D.M. Siringoringo and Y. Fujino, Noncontact Operational Modal Analysis of Structural Members by Laser Doppler Vibrometer, ComputerAided Civil and Infrastructure Engineering 24 (2009), 249-265.

[11] D.M. Siringoringo and Y. Fujino, Experimental study of laser Doppler vibrometer and ambient vibration for vibration-based damage detection, Engineering Structures 28(13) (2006), 1803-1815.

[12] S. Gade, N.B. Moller, N.J. Jacobson and B. Hardonk, Modal Analysis using a Scanning Laser Doppler Vibrometer, IMAC-Proceedings of the 20th International Modal Analysis Conference: Structural Dynamics I and II (2002), 1015-1019.

[13] B. Verrelst, A dynamic walking biped actuated by pleated pneumatic artificial muscles: Basic concepts and control issues. PhD Thesis, Vrije Universiteit Brussel, February 2005. http://lucy.vub.ac.be/publications/PhD_Verrelst.pdf.

[14] Festo Fluidic Muscle DMSP, Festo brochure Fluidic Muscle. Also available at: http://www.festo.com/cat/nl-be_be/data/doc_engb/PDF/ EN/DMSP-MAS_EN.PDF.

[15] Festo Fluidic Musce DMSP-.../MAS... Operating instructions. Festo AG and Co KG, Postfach D-73726 Esslingen, version 0501c.

[16] E. Parloo, P. Guillaume and B. Cauberghe, Maximum likelihood identification of non-stationary operational data, Journal of Sound and Vibration 268 (2003), 971-991.

[17] B. Cauberghe, P. Guillaume, P. Verboven, E. Parloo and S. Vanlanduit, A combined experimental-operational modal analysis approach in the frequency domain. Proceedings of the 21st International Modal Analysis Conference (IMAC XXI), Kissemmee, Florida, USA, 2003.

[18] P. Guillaume, T. De Troyer, C. Devriendt and G. De Sitter, OMAX - a combined experimental-operational modal analysis approach, in: Proceedings of ISMA2006 International Conference on Noise and Vibration Engineering, P. Sas and M. De Munck, eds, Leuven, Belgium, September 2006, pp. 2985-2996. 

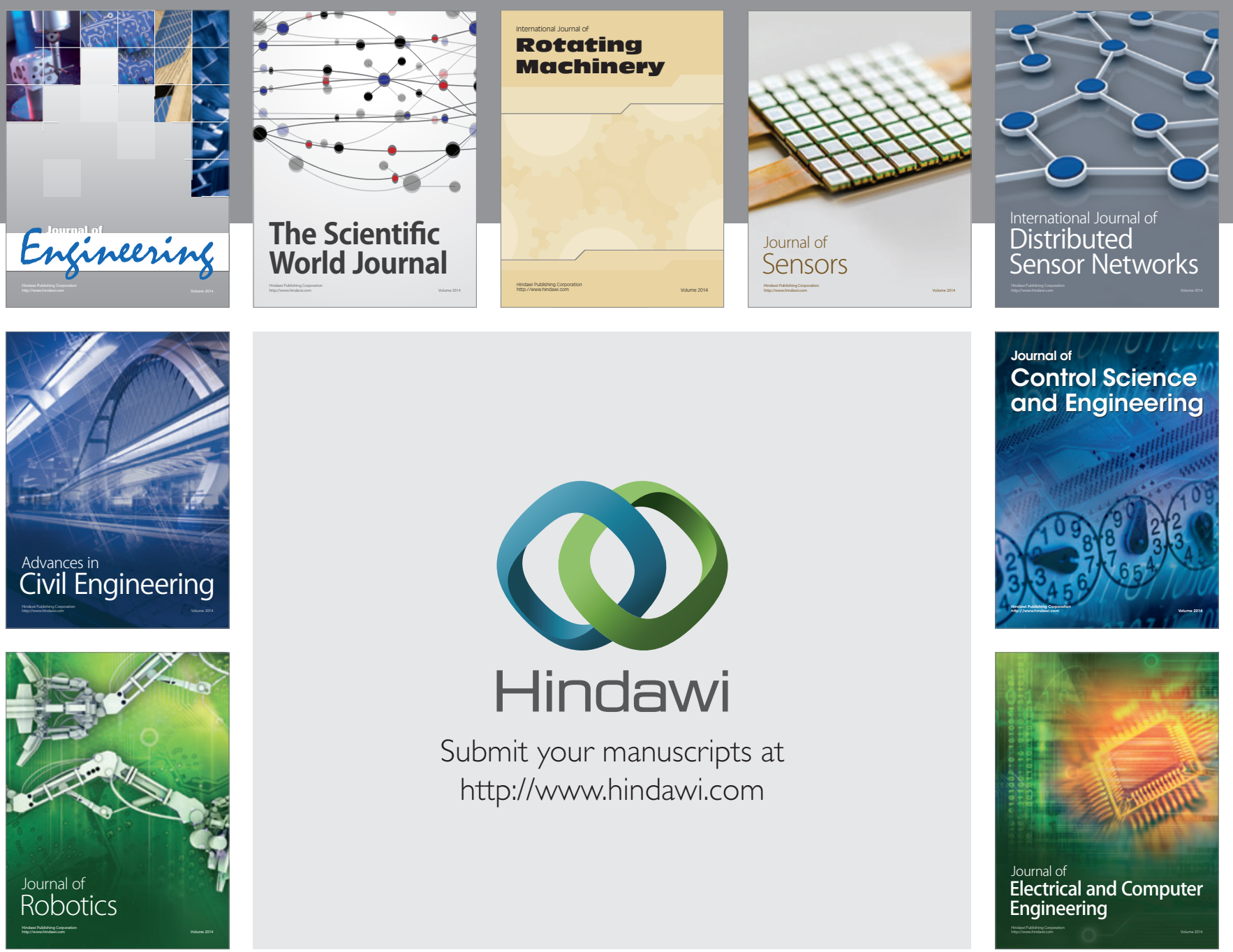

Submit your manuscripts at

http://www.hindawi.com
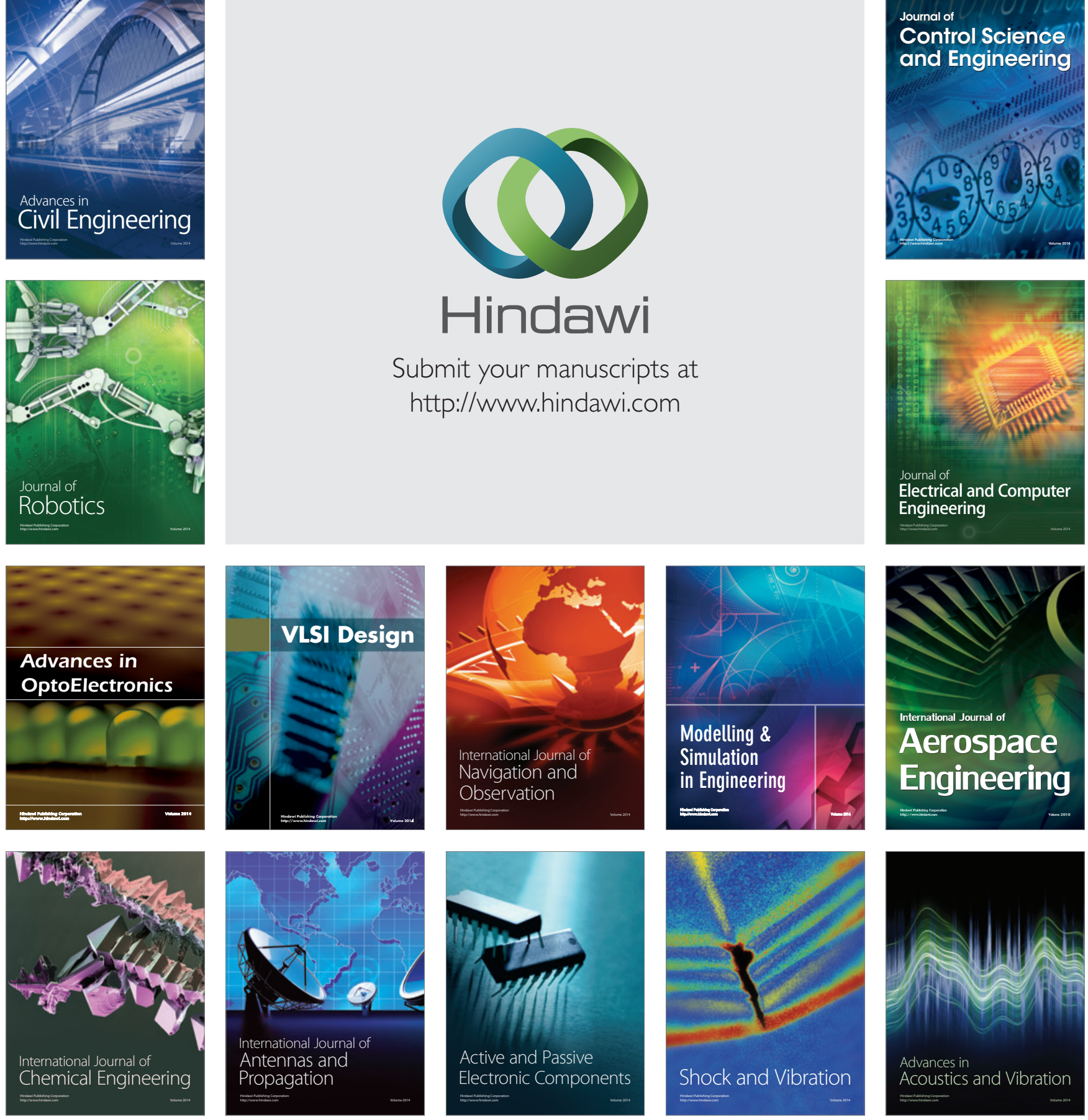